\title{
Emerging roles of RNA methylation in gastrointestinal cancers
}

\author{
Shanshan Xie ${ }^{1,2}$, Wenwen Chen ${ }^{2}$, Kanghua Chen², Yongxia Chang², Feng Yang ${ }^{2}$, Aifu Lin³ , Qiang Shu', \\ Tianhua Zhou ${ }^{2,4^{*}}$ and Xiaoyi Yan ${ }^{2^{*}}$ (D)
}

\begin{abstract}
RNA methylation has emerged as a fundamental process in epigenetic regulation. Accumulating evidences indicate that RNA methylation is essential for many biological functions, and its dysregulation is associated with human cancer progression, particularly in gastrointestinal cancers. RNA methylation has a variety of biological properties, including N6-methyladenosine (m6A), 2-O-dimethyladenosine (m6Am), N1-methyladenosine (m1A), 5-methylcytosine (m5C) and 7-methyl guanosine (m7G). Dynamic and reversible methylation on RNA is mediated by RNA modifying proteins called "writers" (methyltransferases) and "erasers" (demethylases). "Readers" (modified RNA binding proteins) recognize and bind to RNA methylation sites, which influence the splicing, stability or translation of modified RNAs. Herein, we summarize the biological functions and mechanisms of these well-known RNA methylations, especially focusing on the roles of $\mathrm{m} 6 \mathrm{~A}$ in gastrointestinal cancer development.
\end{abstract}

Keywords: RNA methylation, m6A, m6Am, m1A, m5C, Gastrointestinal cancers

\section{Background}

Gastrointestinal (GI) cancers refer to malignant conditions of the GI tract and accessory organs of digestion including liver and pancreas. Globally, GI cancers account for roughly half of all cancer-related deaths [1, 2]. GI cancers have high mortality rates, mainly because of asymptomatic at early-stages, and limited treatment options and poor prognosis at advanced stages. Thus, the identification of robust biomarkers for early-stage and the development of new drugs to treat GI cancers are urgently needed.

Even though modification events in RNA molecules were discovered in the 1950s, our understanding of RNA modification is limited [3-6]. Recently, with the rapid development of transcriptomics technologies, studies about the physiological and pathological function of RNA

\footnotetext{
*Correspondence: tzhou@zju.edu.cn; yanxiaoyi@zju.edu.cn

${ }^{2}$ Department of Cell Biology, Zhejiang University School of Medicine, Hangzhou 310058, China

Full list of author information is available at the end of the article
}

modification come to the forefront. To date, over 170 RNA modifications have been identified, including RNA methylation and pseudouridylation $(\Psi)$. These modifications have been identified to distribute extensively in kinds of RNAs, such as messenger RNAs (mRNAs), ribosomal RNAs (rRNAs), transfer RNAs (tRNAs), small nuclear RNAs (snRNAs) and small nucleolar RNAs (snoRNAs) [7].

Among RNA modifications, RNA methylation is the most well-characterized type. Highly dynamic and reversible methylation on RNA is mediated by a number of proteins, which are called RNA-modifying proteins (RMPs) $[8,9]$. RMPs include "writers" and "erasers" that respectively decorate and remove methylations on RNA respectively, and "readers" that recognize and bind to the methylation sites. The fates of modified RNA depends on the functions of distinct "readers" that may affect their metabolism process (splicing, stability or translation). RNA methylation plays critical roles in diverse physiological processes, and its alterations has been linked to various human cancer [10-16].

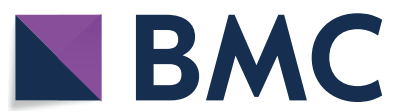

(c) The Author(s) 2020. This article is licensed under a Creative Commons Attribution 4.0 International License, which permits use, sharing, adaptation, distribution and reproduction in any medium or format, as long as you give appropriate credit to the original author(s) and the source, provide a link to the Creative Commons licence, and indicate if changes were made. The images or other third party material in this article are included in the article's Creative Commons licence, unless indicated otherwise in a credit line to the material. If material is not included in the article's Creative Commons licence and your intended use is not permitted by statutory regulation or exceeds the permitted use, you will need to obtain permission directly from the copyright holder. To view a copy of this licence, visit http://creativecommons.org/licenses/by/4.0/. The Creative Commons Public Domain Dedication waiver (http://creativecommons.org/publicdomain/zero/1.0/) applies to the data made available in this article, unless otherwise stated in a credit line to the data. 
In this review, we focus on metabolic regulatory mechanisms and biological functions of RNA methylation. Moreover, we summarize the role of dysregulated m6A modifying proteins in cancer initiation and progression, and discuss the potential of targeting m6A modifying proteins with aberrant expression for therapy.

\section{RNA methylation}

In chemistry, methylation means a form of alkylation that adds a methyl group on a substrate or substitutes the original atom or group [17]. In biological systems, methylation confers to epigenetic alterations to regulate its expression but not affect gene sequence. It can occur in varieties of biomolecules including DNA, RNA and proteins. RNA methylation was first reported as early as the 1950s. Gutman et al. found that N6-methyladenine, N2-methylguanine and 1-methylguanine occurred in the ribonucleic acid hydrolysates in yeast [18]. To date, more than 70 of RNA methylations have been found in diverse kinds of RNA molecules across species. RNA methylation is a reversible post-translational modification that epigenetically affects many biological processes, such as RNA stability, localization, mRNA translation and translocation $[19,20]$. Here, we will introduce some well-known types of RNA methylation, particularly N6-methyladenosine (m6A), 2-O-dimethyladenosine (m6Am), N1-methyladenosine (m1A), 5-methylcytosine (m5C) and 7-methyl guanosine (m7G) (Fig. 1).

\section{m6A modification}

m6A refers to methylation of the adenosine base at the nitrogen-6 position (Fig. 2a). It was originally identified in mRNA in 1974. So far, m6A has been considered as the most abundant internal methylation in mRNA, with around $25 \%$ of mRNA carrying at least one m6A site [3, $21,22]$. In 2012, a transcriptome-wide m6A site mapping reveals that $\mathrm{m} 6 \mathrm{~A}$ sites are present on more than 7600 mRNAs and 300 noncoding RNAs, and are enriched near the stop codons and in the $3^{\prime}$ UTRs of mRNAs of human and mice. $\mathrm{m} 6 \mathrm{~A}$ is also found in rRNA, tRNA and snRNA. Almost $90 \%$ of m6A peaks contain at least one of m6A consensus motifs $[23,24]$. The predominant consensus sequence is "A/G-A/G-methylated A-C-U".

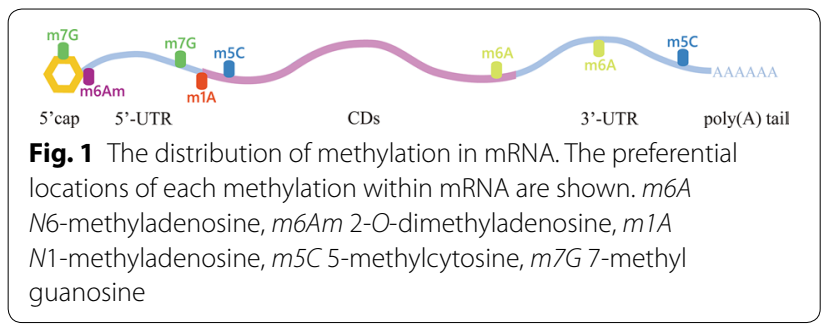

$\mathrm{m} 6 \mathrm{~A}$ is dynamically regulated by specific methyltransferases and demethylases. The most well-characterized methyltransferases is the core methyltransferase complex, which consists of methyltransferase-like 3 (METTL3) and methyltransferase-like 14 (METTL14) (Fig. 2a). METTL3, an $S$-adenosyl-L-methionine (SAM)-binding protein, is identified as a catalytic subunit in this complex, while METTL14 serves as an essential component to facilitate RNA binding [25]. Recent research shows that Wilms tumor 1-associating protein (WTAP) interacts with the METTL3-METTL14, which acts as a third subunit of the complex [26]. Fat mass and obesity-associated protein (FTO) is the first m6A eraser discovered, which catalyze the oxidation of m6A to $N(6)$-hydroxymethyladenosine $(\mathrm{hm} 6 \mathrm{~A})$ as an intermediate modification, and further oxidize hm6A to $N(6)$-formyladenosine [27, 28]. AlkB homolog 5 (ALKBH5), another well-known demethylase, directly removes the methyl group from the methylated adenosine but not oxidative demethylation [29]. Several reader proteins directly recognize and bind m6A-containing mRNAs, including YTH-domaincontaining family proteins (YTHDF1-3, YTHDC1-2) and insulin-like growth factor 2 mRNA-binding proteins (IGF2BP1-3), which is involved in RNA fate decision [12, $30,31]$.

\section{m6Am modification}

m6Am was discovered at the $5^{\prime}$ ends of up to $30 \%$ mRNAs in 1975 [32]. The first nucleotide after an m7G cap can be methylated on the ribose sugar to form 2'-O-methyladenosine (Am) (Fig. 2b). Then, Am could be further methylated at its N6 position to generate m6Am $[33,34]$. A transcriptome-wide m6Am mapping reveals that the $\mathrm{m} 6 \mathrm{Am} / \mathrm{A}$ ratio of total RNA ranges from 0.0036 to $0.0169 \%$ in human tissues, and the m6Am levels show a negative correlation with the corresponding protein expression [35]. More recent studies identify phosphorylated CTD interacting factor 1 (PCIF1) as a mammalian mRNA m6Am methyltransferase [36-38]. In addition, FTO has been reported to convert m6Am to Am in cellular mRNAs $[39,40]$.

\section{m1A modification}

$\mathrm{m} 1 \mathrm{~A}$ is a reversible methylation with the addition of a methyl group and a positive charge at the $\mathrm{N} 1$ position of adenosine, which blocks the Watson-Crick interface, alters RNA secondary structures and protein-RNA interactions [41] (Fig. 2c). The first study on $\mathrm{m} 1 \mathrm{~A}$ in total RNA was back to 1961 [42]. Later, m1A was found in rRNA, tRNA, mRNA and mitochondrial RNAs [43-48]. m1A was highly abundant in tRNA and rRNA, while it displayed a low abundance in mRNA. The $\mathrm{m} 1 \mathrm{~A} / \mathrm{A}$ ratio 


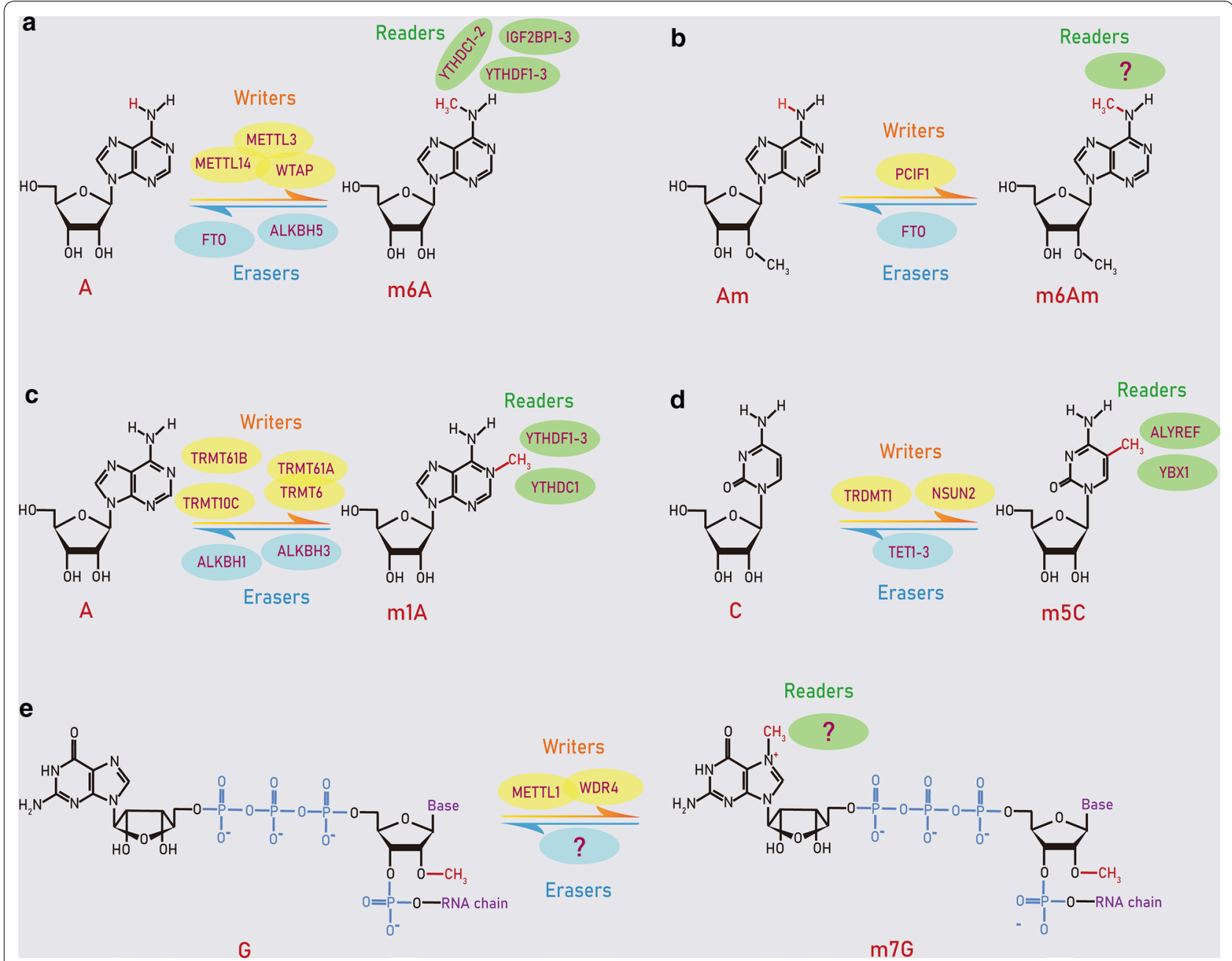

Fig. 2 Molecular composition of RNA methylation. Reversible methylations on RNAs are mediated by RNA modifying proteins called "writers" (yellow), "erasers" (blue). "Readers" (green) are also shown

in mammalian cells is about $0.02 \%$ and up to $0.16 \%$ of mammalian tissues $[45,46]$.

The best-characterized $\mathrm{m}^{1} \mathrm{~A}$ methyltransferase responsible for cytoplasmic tRNAs has two subunits, TRMT6 and TRMT61A in eukaryote [49] (Fig. 2c). TRMT61A has tRNA adenine- $N 1$-methyltransferase catalytic activity, and TRMT6 is important for tRNA binding [50]. In addition, TRMT10C and TRMT61B are known to catalyze $\mathrm{m} 1 \mathrm{~A}$ at positions 9 and 58 in mitochondria tRNAs $[51,52]$. Recent studies find that these tRNA methyltransferases may also catalyze $\mathrm{m} 1 \mathrm{~A}$ in mRNAs [47, 48]. For example, TRMT6/61A writes $\mathrm{m} 1 \mathrm{~A}$ in some mRNAs carrying a GUUCRA tRNA-like motif, whereas TRMT61B and TRMT10C are able to add m1A sites in some mitochondria mRNAs. Similar to m6A, two AlkB family proteins ALKBH1 and ALKBH3 have been reported to demethylate $\mathrm{m} 1 \mathrm{~A}$ sites in RNAs $[46,53]$.
ALKBH1 is an m1A demethylase in cellular tRNAs [53], while ALKBH3 is identified as a demethylase in both mRNAs and tRNAs [46]. A recent study suggests that YTHDF1-3 and YTHDC1, but not YTHDF2, are putative m1A readers [54].

\section{m5C modification}

$\mathrm{m} 5 \mathrm{C}$ occurs in both DNA and RNA by introducing a methyl group in the 5th carbon atom of cytosine [55] (Fig. 2d). The highly abundant $\mathrm{m} 5 \mathrm{C}$ exists in a number of RNAs, including mRNAs, tRNAs, rRNAs and enhancer RNAs (eRNAs). m5C sites in mRNA are generally located in the vicinity of the argonaute-binding regions within the $3^{\prime}$ UTR or near the translational start site $[56,57]$. In eukaryotic, $\mathrm{m} 5 \mathrm{C}$ is introduced by the DNA methyltransferase homolog DNMT2 or the NSUN family members (NSUN1-7) $[56,58]$. NSUN2 catalyzes the m5C in 
mRNAs, or at position 34 of intron-containing tRNA ${ }^{(\mathrm{Leu})}$ (CAA) precursors [56, 59]. The major RNA targets of DNMT2 is tRNA $^{\text {Asp }}$, tRNA ${ }^{\text {Gly }}$ and tRNA ${ }^{\text {Val }}$ [60]. The teneleven translocation (Tet) family proteins (TET1-3) are $\mathrm{Fe}(\mathrm{II})^{-}$and 2-oxoglutarate-dependent dioxygenases to demethylate $\mathrm{m} 5 \mathrm{C}$ sites in mammalian RNAs [61]. These TET enzymes possess the catalytic activity to induce the formation of 5-hydroxymethylcytidine in RNAs. Additionally, the mRNA export adaptor Aly/REF export factor (ALYREF, also known as THOC4) and Y-box binding protein 1 (YBX1) have been reported as an $\mathrm{m} 5 \mathrm{C}$ readers for mRNAs, which recognizes and binds to $\mathrm{m} 5 \mathrm{C}$-modified mRNAs [57, 62].

\section{m7G modification}

Most eukaryotic mRNAs have a methyl group and a positive charge at the N7 position of the terminal guanosine at its $5^{\prime}$ cap (Fig. 2e). m7G is present at not only mRNA caps, but also some internal positions within mRNAs, tRNAs and rRNAs [63, 64]. The most well-characterized enzyme mediating internal $\mathrm{m} 7 \mathrm{G}$ methylation is METTL1, which works together with its co-factor WD repeat domain 4 (WDR4) [65].

\section{Biological functions of RNA methylation}

Recently, the rapid development of transcriptome-wide profiling of diverse RNA methylations, along with the identification of the corresponding writers, erasers and readers, enable researchers to reveal the regulatory roles of RNA methylation in RNA metabolism and a wide range of cellular and biological processes.

\section{The roles of RNA methylations in RNA metabolism}

RNA methylations appear to function through nearly all steps of RNA metabolism, including RNA capping, splicing, stability, translation, and nuclear exportation (Fig. 3). The protein complex DXO/Dom3Z can decap the unmethylated cap and degrade the remaining part of pre-RNA [66]. Depletion of the m6A writer WTAP leads to abnormal mRNA isoforms [26]. It has been reported that $\mathrm{m} 6 \mathrm{~A}$ is enriched in exonic regions flanking $5^{\prime}$ - and $3^{\prime}$-splice sites, where are the binding regions of mRNA splicing regulatory serine/arginine-rich protein 2 (SRSF2). Knockdown of the m6A eraser FTO results in elevated m6A level in the exonic regions, and enhanced mRNA binding ability of SRSF2, which lead to an increased inclusion of target exons [67]. These results indicate that RNA methylation may be involved in mRNA capping and splicing.

Nuclear export is an essential step for mRNA to ensure its translation in the cytoplasm. Knockdown of the m6A easer ALKBH5 induces poly $(\mathrm{A})^{+}$RNA retention in the nucleus [29]. The $\mathrm{m} 5 \mathrm{C}$ reader ALYREF depletion also

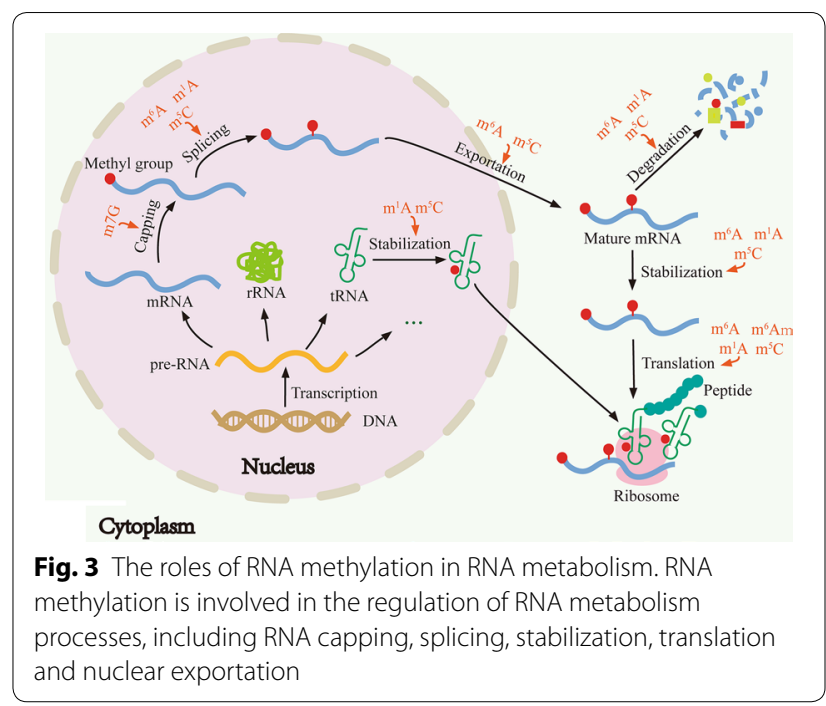

increases the nuclear retention of $\mathrm{m} 5 \mathrm{C}$-decorated mRNA [57]. These studies suggest that RNA methylation plays an important role in RNA nuclear export.

The dynamic synthesis and degradation of mRNA maintains an acute amount of mRNA in different contexts. For example, METTL3 knockdown decreases the m6A modification of cytokine signaling 2 (SOCS2) mRNA, leading to its degradation [68]. In addition, YTHDF2-bound mRNAs will translocate from translatable pool to mRNA decay sites (processing bodies) [12]. ALKBH1 (an m1A eraser) depletion increases the cellular level of tRNA ${ }^{\mathrm{iMet}}$, suggesting that m1A modification is important for tRNA stability [53]. These data provide a strong connection between RNA methylation and mRNA stability. The m6A reader YTHDF1 has been found to interact with ribosomes and initiation factors, and increase cellular translational output, which is a piece of evidence for m6A function in translational regulation [69]. NSUN2-mediated $\mathrm{m} 5 \mathrm{C}$ methylation inhibits the translation of the cyclin-dependent kinase inhibitor p27KIP1 [70]. Furthermore, recent ribosome profiling analyses suggest that both $\mathrm{m} 6 \mathrm{Am}$ and $\mathrm{m} 1 \mathrm{~A}$ play important roles in regulating translation $[37,48]$.

\section{The roles of RNA methylations in biological processes}

Considering the diverse regulation of RNA methylation on RNA fate, it would not be surprising that RNA methylation regulates many cellular and developmental processes. m6A modification has been documented to be important for nervous system development. Mettl3 conditional knockout mice display the enhanced apoptosis of newborn cerebellar granule cells in the external granular layer, and lower levels of m6A modification in brain mRNA with aberrant RNA splicing and stability [71]. 
Postnatal deletion of Mettl3 in the mouse hippocampus exhibit no significant morphological change but reduced memory consolidation ability [72]. Embryonic cortical radial glia cells in mice deleted of Mettl3 or Mettl4 manifests a prolonged cell cycle [73]. Additionally, FTO deficiency in mice decreases the proliferation and neuronal differentiation of adult neural stem cells, consequently leading to impaired learning and memory [74].

m6A modification is also important for germline development in mice. The ablation of mouse METTL3 inhibits spermatogonial differentiation and blocks the initiation of meiosis in germ cells [75]. The deficiency of ALKBH5 shows apoptosis of spermatocytes in pachytene and metaphase-stage, resulting in aberrant spermiogenesis and low number and poor quality of spermatozoa in the male mice [29]. YTHDC2 is highly expressed in mouse testes, and the spermatocytes of $Y t h d c 2$ knockout mice fail to undergo pachytene, leading to defects in spermatocyte development [76].

RNA methylation also influences other fundamental physiological processes in development. Deletion of drosophila MTEEL3 homolog Ime4 abolishes the m6A modification of the sex determination factor sex-lethal $(S x l)$, leading to $\mathrm{X}$ inactivation failure and improper sex determination [11, 77]. Zebrafish embryos that lack Mettl3 show a decrease in hematopoietic stem/progenitor cell numbers and defects in endothelial-to-hematopoietic transition [78]. Ythdf2-deficiency zebrafish embryos display developmental delay because of impaired decay of m6A-modified maternal RNAs during the maternal-tozygotic transition [79].

\section{Roles of $\mathbf{m 6 A}$ in $\mathrm{Gl}$ cancers}

As mentioned above, RNA methylation has been implicated in diverse biological processes. There is no doubt that its dysregulation is highly associated with tumor initiation and progression. Accumulating evidence has shown that dysregulation of RNA methylation in cancer development, and the most well-studied one is m6A. $\mathrm{m} 6 \mathrm{~A}$ involves in cancer progression often through the actions of writers and easers that alter the abundance of $\mathrm{m} 6 \mathrm{~A}$ in the mRNA of oncogenes or tumor-suppressive genes and then the readers that recognize the modified sites to further upregulate oncogene or downregulate tumor-suppressive gene expression.

Many studies have shown that m6A modification plays a crucial role in various cancers. For example, the master methylase of m6A METTL3 has been reported to involve in many steps of RNA processing and the oncogenic pathway in different cancer types. In glioblastoma, METTL3 facilitates the maintenance and radiation resistance of glioma stem-like cells via increasing the m6A modification of SRY-box transcription factor 2
(SOX2), which enhances the stability and protein expression of SOX2 mRNA [80]. In non-small cell lung cancer, METTL3 triggers treatment resistance and metastasis of lung cancer cells by promoting the translation efficiency and stability of yes-associated protein mRNA [81]. METTL3 is also overexpressed in melanoma, which enhances the invasion and migration of human melanoma cells through increasing the expression of matrix metallopeptidase 2 [82]. Meanwhile, many other RNA modifying proteins are also reported to be contributed to the initiation and progression of human cancers. Herein, we mainly focus on the biological roles of aberrant m6A modification in gastrointestinal cancers, including gastric cancer, colorectal cancer, liver cancer and pancreatic cancer (Table 1).

\section{Gastric cancer}

Gastric cancer develops from the lining of the stomach. More than 950,000 patients are diagnosed as gastric cancer, and about 720,000 patients died from gastric cancer every year worldwide [83]. It is usually divided into intestinal, diffuse and mixed histological subtypes according to Lauren classification [84]. METTL3 has been reported as an important player in gastric cancer development. Wang et al. [85] reported that the levels of m6A RNA and METTL3 were increased in gastric cancer tissues. METTL3, which expression is correlated with poor prognosis, promotes gastric cancer cell proliferation and liver metastasis by stimulating m6A modification of heparin binding growth factor (HDGF) mRNA. In addition, the m6A reader IGF2BP3 recognizes and binds to HDGF mRNA to enhance its stability. The increased HDGF then activates GLUT4 and ENO2 to induce glycolysis in gastric cancer cells. Yue et al. showed that METTL3 was upregulated in gastric cancer tissues, and was critical for epithelial-mesenchymal transition (EMT) process of gastric cancer cells and tumor metastasis [86]. Zinc finger MYM-type containing 1 (ZMYM1) mRNA is methylated by METTL3 and interacts with the m6A reader protein $\mathrm{HuR}$ to increase its stabilization. ZMYM1 binds to and represses the promoter of E-cadherin and then facilitates EMT program and the consequent metastasis of gastric cancer cells. Moreover, another two groups also found that METTL3 promoted the proliferation and mobility of gastric cancer cells [87, 88]. Zhang et al. [89] revealed that ALKBH5 promoted gastric cancer cell invasion by decreasing the methylation of the lncRNA nuclear paraspeckle assembly transcript 1 (NEAT1).

\section{Colorectal cancer}

Colorectal cancer develops from one or a few small polyps inside the colon, and gradually develops into mature colorectal cancers. More than 1.8 million new colorectal 
Table 1 RNA modifying proteins of $\mathbf{m 6 A}$ in gastrointestinal cancers

\begin{tabular}{|c|c|c|c|c|c|}
\hline Cancer types & RMPs & Expression & Biological FUNCTIONS & Targets & References \\
\hline \multirow[t]{3}{*}{ Gastric cancer (GC) } & METTL3 & Up & Promote GC cell proliferation and liver metastasis & HDGF mRNA & {$[86]$} \\
\hline & & & Facilitate the EMT program and matastasis & ZMYM1 mRNA & {$[87,88]$} \\
\hline & $\mathrm{ALKBH} 5$ & Up & Promote GC cell invasion and migration & NEAT1 & {$[89]$} \\
\hline \multirow[t]{6}{*}{ Colorectal cancer (CRC) } & METTL3 & Up & $\begin{array}{l}\text { Promote CRC cell self-renewal and colorectal tumor growth } \\
\text { and metastasis }\end{array}$ & SOX2 mRNA & {$[91]$} \\
\hline & & & Promote CRC metastasis & pri-miR-1246 & {$[91]$} \\
\hline & & Down & Inhibit CRC cell proliferation, invasion and migration & Unknown & {$[92]$} \\
\hline & & & $\begin{array}{l}\text { Inhibit CRC cell growth and invasion, tumor growth and } \\
\text { metastasis }\end{array}$ & SOX4 mRNA & {$[94]$} \\
\hline & & & $\begin{array}{l}\text { Inhibit CRC cell proliferation and invasion, tumorigenicity and } \\
\text { metastasis }\end{array}$ & XIST mRNA & {$[95]$} \\
\hline & YTHDF1 & Up & $\begin{array}{l}\text { Promote CRC cell proliferation, drug sensitivity and tumor } \\
\text { growth }\end{array}$ & Unknown & {$[96,97]$} \\
\hline \multirow[t]{8}{*}{ Liver cancer (HCC) } & METTL3 & Up & $\begin{array}{l}\text { Promote HCC cell proliferation, tumorigenicity and lung } \\
\text { metastasis }\end{array}$ & sOCS2 mRNA & {$[68]$} \\
\hline & METTL14 & Down & Inhibit tumor metastasis & microRNA 126 & {$[68,98]$} \\
\hline & & & & DGCR8 mRNA & [99] \\
\hline & WTAP & Up & Enhance HCC cell proliferation and tumor progression & ETS1 mRNA & {$[99]$} \\
\hline & KIAA1429 & Up & Promote the proliferation and invasion of HCC cells & ID2 mRNA & {$[100]$} \\
\hline & $\mathrm{FTO}$ & Up & Promote the proliferation and tumor growth & PKM2 mRNA & {$[101]$} \\
\hline & & Down & $\begin{array}{l}\text { Increase the apoptosis of ICC cells and reduce resistance to } \\
\text { cisplatin treatment }\end{array}$ & Unknown & {$[102]$} \\
\hline & YTHDF2 & Down & $\begin{array}{l}\text { Enhance inflammation, vascular reconstruction and metastatic } \\
\text { progression of HCC cells }\end{array}$ & IL-11, SERPINE2 mRNA & {$[103]$} \\
\hline \multirow[t]{3}{*}{ Pancreatic cancer (PC) } & METTL3 & Up & Promote PC cell proliferation and invasion & Unknown & {$[105,106]$} \\
\hline & $\mathrm{ALKBH} 5$ & Down & Inhibit PC cell motility & KCNK15-AS1 & {$[107]$} \\
\hline & & & $\begin{array}{l}\text { Inhibit PDAC cell proliferation, migration, and invasion both } \\
\text { in vitro and in vivo }\end{array}$ & WIF-1mRNA & [108] \\
\hline
\end{tabular}

cancer cases and 881,000 deaths are estimated to occur in 2018 [1]. Analyses of the Cancer Genome Atlas (TCGA) datasets show that the highly expression of METTL3 mRNA in the metastatic colorectal cancer tissue is positively associated with poor prognosis of patients [90]. Knockdown of METTL3 suppresses the self-renewal, stem cell frequency and migration of colorectal cancer cells in vitro, and inhibits the colorectal tumor growth and metastasis in vivo. Mechanistically, METTL3 targets to SRY (sex determining region Y)-box 2 (SOX2) transcripts. The methylated SOX2 mRNAs are recognized and stabilized by the m6A reader IGF2BP2. Peng et al. [91] also reported that upregulated METTL3 facilitated colorectal cancer metastasis by methylation of pri-miR-1246, further influencing the downstream miR-1246/SPRED2/MAPK signaling axis. Deng et al. [92] found that knockdown of METTL3 promoted colorectal cancer cell proliferation and invasion possibly by increasing the phosphorylation of p38 and ERK. Interestingly, a strong correlation between METTL3 and ${ }^{18} \mathrm{~F}$ FDG uptake is observed in colorectal cancer patients [93]. Furthermore, METTL3 modified m6A in HK2 and
SLC2A1 transcripts to stabilize these two genes and activate the glycolysis pathway. m6A-mediated HK2 and SLC2A1 (GLUT1) stabilization depends on the reader protein IGF2BP2 or IGF2BP2/3, respectively. METTL14 expression is significant decreased in colorectal cancer and its lower expression is correlated with poor overall survival of patients [93]. Functionally, METTL14 knockdown leads to enhanced colorectal cancer cell growth and invasion in vitro and tumor growth and metastasis in vivo [94]. Mechanistically, METTL14 depletion results in decreased m6A modification and elevated expression of SRY-related high-mobility-group box 4 (SOX4) mRNA in a YTHDF2-dependent manner. Additionally, Zheng's group also found that loss of METTL14 is associated with poor prognosis of colorectal cancer patients [94]. Knockdown of METTL14 promotes proliferation and invasion of colorectal cancer cells in vitro and enhanced tumorigenicity and metastasis in vivo. Furthermore, METTL14 depletion abolishes m6A level of XIST and attenuates its expression. YTHDF2 could recognized the m6A-methylated XIST to mediate its degradation [95]. Additionally, the m6A reader YTHDF1 was also found to 
be upregulated in colorectal cancer and correlated with poor prognosis of patients [96]. Furthermore, knockdown of YTHDF1 inhibits colorectal cancer cell proliferation, colonosphere formation, anti-cancer drug sensitivity and murine xenograft tumor growth in mice [97].

\section{Liver cancer}

Liver cancer is predicted to be the fourth leading cause of cancer death worldwide, with about 841,000 new cases and 782,000 deaths annually [1]. The most common type of liver cancer is hepatocellular carcinoma (HCC), which is developed from the transformed hepatocytes and accounts for more than $75 \%$ of liver cancer cases [2]. The level of METTL3 mRNA is highly expressed in HCC, and its upregulation is correlated with poor prognosis of patients [68]. METTL3 has been further documented to not only induce $\mathrm{HCC}$ cell proliferation, colony formation and migration in vitro, but also promotes HCC tumorigenicity and lung metastasis by stabilizing suppressor of SOCS2 mRNA in a YTHDF2-dependent pathway. Unlike the significant increase of METTL3, METTL14 expression in HCC tissues shows a mild increase, and promotes $\mathrm{HCC}$ cell proliferation, colony formation and migration in vitro [68]. In contrast, $\mathrm{Ma}$ et al. [98] reported that METTL14 was downregulated in HCC tissues, and knockdown of METTL14 enhances tumor metastasis. METTL14 was further showed to bind to the microprocessor protein DGCR8 mRNA and increase microRNA-126 in an m6A-dependent manner. WTAP also enhances the capability of HCC cell proliferation and tumor progression via the HuR-ETS1-p21/ p27 axis [99]. Moverover, Cheng et al. [100] showed that another m6A writer KIAA1429 was upregulated in HCC tissues, and it promoted the proliferation and invasion of HepG2 cells by stabilizing the inhibitor of DNA binding 2 (ID2) mRNA. The m6A easer FTO is upregulated in HCC tissues, which correlates with poor prognosis of HCC patients [101]. FTO facilitates the proliferation and in vivo tumor growth via demethylating of $P K M 2 \mathrm{mRNA}$ and enhancing its translation efficiency. In contrast, FTO is found to be downregulated in intrahepatic cholangiocarcinoma (ICC). Loss of FTO in ICC is associated with tumor aggressiveness and poor prognosis. Suppression of FTO reduces the apoptosis of ICC cells and increases resistance to cisplatin treatment [102]. The expression of m6A reader YTHDF2 is decreased in HCC tissues and its downregulation is correlated with poor classification and prognosis of HCC patients [103]. YTHDF2 processes the decay of m6A-containing interleukin 11 and serpin family $\mathrm{E}$ member 2 transcripts to attenuate inflammation, vascular reconstruction and metastatic progression of HCC cells.

\section{Pancreatic cancer}

Pancreatic cancer is the seventh leading cause of cancer death, with over 1,000,000 new cases and 65,000 deaths estimated globally [1]. Pancreatic ductal adenocarcinoma (PDAC) is the most lethal subtype of pancreatic cancer and accounts for more than $80 \%$ of pancreatic cancer patients [104]. METTL3 has been found to be highly expressed in pancreatic tumor tissues. METTL3depleted pancreatic cancer cells display a decreased ability of cell proliferation and invasion, and an increased sensitivity to the treatment of anticancer reagents and irradiation $[105,106]$. Recent study showed that ALKBH5 was downregulated in pancreatic cancer cells, and involved in the cell motility by demethylating the lncRNA KCNK15-AS1 [107]. In addition, Shimamoto's group showed that decreased ALKBH5 levels predicts poor clinical outcome of patients with pancreatic ductal adenocarcinoma (PDAC) [108]. Silencing ALKBH5 increases PDAC cell proliferation, migration, and invasion both in vitro and in vivo. Mechanistically, ALKBH5 suppresses Wnt signaling and its downstream targets though demethylation of m6A-modified WIF-1 transcripts.

\section{Targeting RNA modifying proteins for cancer treatment}

Given that m6A modifying proteins play important roles in so many malignant behaviors of cancer cell, they could be used as potential therapeutic targets for cancer treatment. Some FTO inhibitors have been developed by different research groups to increase the $\mathrm{m} 6 \mathrm{~A}$ abundance [109, 110]. MA2, CS1 and CS2 have been shown to inhibit the growth and self-renewal of glioblastoma stem cells and leukemia stem cells, respectively [111, 112]. Another two promising FTO inhibitors, namely FB23 and FB23-2, which directly bind to FTO and selectively suppress the m6A demethylase activity of FTO, dramatically enhance the apoptosis of acute myeloid leukemia (AML) cells [113]. Peng et al. [114] showed another compound entacapone, a catechol-O-methyltransferase inhibitor, as an inhibitor of FTO. Furthermore, citrate and IOX3 may be potential inhibitors of another m6A demethylase, ALKBH5, which also maintains tumorigenicity of several types of cancer cells $[115,116]$. In addition, the small molecule inhibitor BTYNB (2-\{[(5-bromo-2-thienyl) methylene]amino\} benzamide) has been demonstrated to show potently anti-proliferative effects in leukemia cells [117], ovarian cancer cell and melanoma cells by disrupting the reader protein IGF2BP1 [118]. Thus, researches on the functional mechanism of RNA modifying proteins will provide clues to the development of corresponding inhibitors for human cancer treatment. Moreover, studies 
focus on the investigation of RNA demethylase inhibitors are strongly needed in the near future.

\section{Conclusion and future perspectives}

During the past 5 years, researchers have found that RNA modification are implicated in mRNA splicing, stability, translation, nuclear export and a wide range of biological processes. RNA modification and RNA-modifying proteins are often found to be aberrant expressed in tumor tissues. The changes of RNA modifications and their RNA-modifying proteins in cancer affects almost every aspect of RNA fate and is context-dependent. These RNA-modifying proteins linked to cancer are important regulators of cellular events by regulating RNA metabolism and expression of certain gene required for cancer cell proliferation, transformation, invasion, and other malignant behaviors. Studies on cancer epitranscriptomics focused on the complex context and heterogeneity of tumor could help us to better understand the exact roles of RNA modifications and their enzymes, which is benefit for exploring RNA modifying proteins as potential therapeutic targets.

Even though emerging data show the potential roles of RNA methylation in gastrointestinal cancer development, some important questions still remain to be addressed. First, how does the same RNA modifying protein selectively catalyze the different target transcripts in different cancer types. This may be attributed to the different upstream regulator of RNA modifying protein upon the specific cellular context in different cancers. Second, the inconsistent findings of the same RNA modifying protein were reported in the same cancer. One possible interpretation is that our current hypothesis is only based on individual cohorts with a relatively limited number of cancer patients with different genetic background and environmental exposure. Third, although a number of new types of RNA methylation in human have been identified until now, their functions in GI cancers need to be further investigated in the future.

\footnotetext{
Abbreviations

ALYREF: Aly/REF export factor; Am: 2'-O-Methyladenosine; ALKBH5: AlkB homolog 5; EMT: Epithelial-mesenchymal transition; eRNAs: Enhancer RNAs; FTO: Fat mass and obesity-associated protein; GI: Gastrointestinal; Hm6A: N(6)Hydroxymethyladenosine; HCC: Hepatocellular carcinoma; HDGF: Heparin binding growth factor; ID2: DNA binding 2; IGF2BP: Insulin-like growth factor 2 mRNA-binding proteins; m1A: N1-Methyladenosine; m5C: 5-Methylcytosine; m6A: N6-Methyladenosine; m6Am: 2-O-Dimethyladenosine; m7G: 7-Methyl guanosine; METTL3: Methyltransferase-like 3; METTL14: Methyltransferase-like 14; mRNAs: Messenger RNAs; NEAT1: Nuclear paraspeckle assembly transcript 1; PCIF1: Phosphorylated CTD interacting factor 1; PDAC: Pancreatic ductal adenocarcinoma; RMPs: RNA-modifying proteins; rRNAs: Ribosomal RNAs; SAM: S-Adenosyl-L-methionine; snRNAs: Small nuclear RNAs; snoRNAs: Small nucleolar RNAs; SOCS2: Cytokine signaling 2; SRSF2: Serine/arginine-rich protein 2; Sxl: Sex determination factor sex-lethal; SOX2: SRY (sex determining region Y)-box 2; tRNAs: Transfer RNAs; Tet: Ten-eleven translocation; TCGA
}

:The Cancer Genome Atlas; WDR4: WD repeat domain 4; WTAP: Wilms tumor 1-associating protein; YBX1:Y-box binding protein 1; YTHDF: YTH-domaincontaining family proteins; ZMYM1: Zinc finger MYM-type containing 1.

\section{Acknowledgements}

Not applicable.

\section{Authors' contributions}

SX wrote the manuscript; WC, KC, YC and FY contributed in literature review and images; AL and QS modified the manuscript. XY and TZ reviewed and edited the manuscript. All authors read and approved the final manuscript.

\section{Funding}

This review article was supported by the Natural Scientific Foundation of China (31620103911 and 31571446); Young Scientists Fund (31801132).

\section{Availability of data and materials}

Not applicable.

\section{Ethics approval and consent to participate}

Not applicable.

\section{Consent for publication}

Not applicable.

\section{Competing interests}

The authors declare that they have no competing interests.

\section{Author details}

${ }^{1}$ The Children's Hospital, Zhejiang University School of Medicine, Hangzhou 310052, China. ${ }^{2}$ Department of Cell Biology, Zhejiang University School of Medicine, Hangzhou 310058, China. ${ }^{3}$ MOE Laboratory of Biosystem Homeostasis and Protection, College of Life Sciences, Zhejiang University, Hangzhou 310058, China. ${ }^{4}$ Department of Molecular Genetics, University of Toronto, Toronto, ON, Canada.

Received: 8 June 2020 Accepted: 26 November 2020

Published online: 07 December 2020

\section{References}

1. Bray F, Ferlay J, Soerjomataram I, Siegel RL, Torre LA, Jemal A. Global cancer statistics 2018: GLOBOCAN estimates of incidence and mortality worldwide for 36 cancers in 185 countries. CA Cancer J Clin. 2018;68(6):394-424.

2. Rahib L, Smith BD, Aizenberg R, Rosenzweig AB, Fleshman JM, Matrisian LM. Projecting cancer incidence and deaths to 2030: the unexpected burden of thyroid, liver, and pancreas cancers in the United States. Cancer Res. 2014;74(11):2913-21.

3. Desrosiers R, Friderici K, Rottman F. Identification of methylated nucleosides in messenger RNA from Novikoff hepatoma cells. Proc Natl Acad Sci USA. 1974;71(10):3971-5.

4. Adams JM, Cory S. Modified nucleosides and bizarre $5^{\prime}$-termini in mouse myeloma mRNA. Nature. 1975;255(5503):28-33.

5. Dubin DT, Taylor RH. The methylation state of poly A-containing messenger RNA from cultured hamster cells. Nucleic Acids Res. 1975;2(10):1653-68.

6. Perry RP, Kelley DE, Friderici K, Rottman F. The methylated constituents of $L$ cell messenger RNA: evidence for an unusual cluster at the $5^{\prime}$ terminus. Cell. 1975;4(4):387-94.

7. Boccaletto P, Machnicka MA, Purta E, Piatkowski P, Baginski B, Wirecki TK, de Crécy-Lagard V, Ross R, Limbach PA, Kotter A, et al. MODOMICS: a database of RNA modification pathways. 2017 update. Nucleic Acids Res. 2018;46(D1):D303-7.

8. Roundtree IA, Evans ME, Pan T, He C. Dynamic RNA modifications in gene expression regulation. Cell. 2017;169(7):1187-200.

9. Patil DP, Pickering BF, Jaffrey SR. Reading m6A in the transcriptome: m6A-binding proteins. Trends Cell Biol. 2018;28(2):113-27.

10. Warda AS, Kretschmer J, Hackert P, Lenz C, Urlaub H, Höbartner C, Sloan KE, Bohnsack MT. Human METTL16 is a $N^{6}$-methyladenosine $\left(m^{6} \mathrm{~A}\right)$ 
methyltransferase that targets pre-mRNAs and various non-coding RNAs. EMBO Rep. 2017;18(11):2004-14.

11. Haussmann IU, Bodi Z, Sanchez-Moran E, Mongan NP, Archer N, Fray RG, Soller $\mathrm{M} . \mathrm{m}^{6} \mathrm{~A}$ potentiates $\mathrm{S} x \mathrm{l}$ alternative pre-mRNA splicing for robust Drosophila sex determination. Nature. 2016;540(7632):301-4.

12. Wang X, Lu Z, Gomez A, Hon GC, Yue Y, Han D, Fu Y, Parisien M, Dai Q, Jia $\mathrm{G}$, et al. N6-Methyladenosine-dependent regulation of messenger RNA stability. Nature. 2014;505(7481):117-20.

13. Shi H, Wang X, Lu Z, Zhao BS, Ma H, Hsu PJ, Liu C, He C. YTHDF3 facilitates translation and decay of $N^{6}$-methyladenosine-modified RNA. Cell Res. 2017;27(3):315-28.

14. Yang $X$, Yang $Y$, Sun $B F$, et al. 5-Methylcytosine promotes mRNA export-NSUN2 as the methyltransferase and ALYREF as an $m^{5} \mathrm{C}$ reader. Cell Res. 2017;27(5):606-25.

15. Sun T, Wu R, Ming L. The role of m6A RNA methylation in cancer. Biomed Pharmacother. 2019;112:108613

16. Chen $\mathrm{XY}$, Zhang J, Zhu JS. The role of $\mathrm{m}^{6} \mathrm{~A}$ RNA methylation in human cancer. Mol Cancer. 2019;18(1):103.

17. Thauer RK. Biochemistry of methanogenesis: a tribute to Marjory Stephenson. 1998 Marjory Stephenson Prize Lecture. Microbiology (Reading). 1998;144(Pt 9):2377-406.

18. Adler $M$, Weissmann $B$, Gutman AB. Occurrence of methylated purine bases in yeast ribonucleic acid. J Biol Chem. 1958;230(2):717-23.

19. Ji L, Chen X. Regulation of small RNA stability: methylation and beyond. Cell Res. 2012;22(4):624-36.

20. Wang $X, \mathrm{He} C$. Reading RNA methylation codes through methyl-specific binding proteins. RNA Biol. 2014;11(6):669-72.

21. Perry RP, Kelley DE, LaTorre J. Synthesis and turnover of nuclear and cytoplasmic polyadenylic acid in mouse L cells. J Mol Biol. 1974;82(3):315-31.

22. Meyer KD, Jaffrey SR. Rethinking m6A readers, writers, and erasers. Annu Rev Cell Dev Biol. 2017;33:319-42.

23. Meyer KD, Saletore Y, Zumbo P, Elemento O, Mason CE, Jaffrey SR. Comprehensive analysis of mRNA methylation reveals enrichment in $3^{\prime}$ UTRs and near stop codons. Cell. 2012;149(7):1635-46.

24. Dominguez D, Freese P, Alexis MS, Su A, Hochman M, Palden T, Bazile C, Lambert NJ, Van Nostrand EL, Pratt GA, et al. Sequence, structure, and context preferences of human RNA binding proteins. Mol Cell. 2018;70(5):854-67.

25. Liu J, Yue Y, Han D, Wang X, Fu Y, Zhang L, Jia G, Yu M, Lu Z, Deng X, et al. A METTL3-METTL14 complex mediates mammalian nuclear RNA N6-adenosine methylation. Nat Chem Biol. 2014;10(2):93-5.

26. Ping XL, Sun BF, Wang L, Xiao W, Yang X, Wang WJ, Adhikari S, Shi Y, LV $Y$, Chen YS, et al. Mammalian WTAP is a regulatory subunit of the RNA N6-methyladenosine methyltransferase. Cell Res. 2014;24(2):177-89.

27. Fu Y, Zhao X, Dai Q, Zheng G, Yang Y, Yi C, Lindahl $T$, Pan T, Yang YG, He C, et al. N6-Methyladenosine in nuclear RNA is a major substrate of the obesity-associated FTO. Nat Chem Biol. 2011;7(12):885-7.

28. Fu Y, Jia G, Pang X, Wang RN, Wang X, Li CJ, Smemo S, Dai Q, Bailey KA, Nobrega MA, et al. FTO-mediated formation of N6-hydroxymethyladenosine and N6-formyladenosine in mammalian RNA. Nat Commun. 2013:4:1798.

29. Zheng G, Dahl JA, Niu Y, Fedorcsak P, Huang CM, Li CJ, Vågbø CB, Shi $\mathrm{Y}$, Wang WL, Song SH, et al. ALKBH5 is a mammalian RNA demethylase that impacts RNA metabolism and mouse fertility. Mol Cell. 2013;49(1):18-29.

30. Dominissini D, Moshitch-Moshkovitz S, Schwartz S, Salmon-Divon M, Ungar L, Osenberg S, Cesarkas K, Jacob-Hirsch J, Amariglio N, Kupiec $M$, et al. Topology of the human and mouse m6A RNA methylomes revealed by m6A-seq. Nature. 2012;485(7397):201-6.

31. Huang $H$, Weng H, Sun W, Qin X, Shi H, Wu H, Zhao BS, Mesquita A, Liu C, Yuan CL, et al. Recognition of RNA N6-methyladenosine by IGF2BP proteins enhances mRNA stability and translation. Nat Cell Biol. 2018;20(3):285-95.

32. Wei C, Gershowitz A, Moss B. N6, O2'-dimethyladenosine a novel methylated ribonucleoside next to the $5^{\prime}$ terminal of animal cell and virus mRNAs. Nature. 1975;257(5523):251-3.
33. Bélanger F, Stepinski J, Darzynkiewicz E, Pelletier J. Characterization of hMTr1, a human Cap1 2'-O-ribose methyltransferase. J Biol Chem. 2010;285(43):33037-44.

34. Werner M, Purta E, Kaminska KH, Cymerman IA, Campbell DA, Mittra B, Zamudio JR, Sturm NR, Jaworski J, Bujnicki JM. 2'-O-Ribose methylation of cap2 in human: function and evolution in a horizontally mobile family. Nucleic Acids Res. 2011;39(11):4756-68.

35. Liu J, Li K, Cai J, Zhang M, Zhang X, Xiong X, Meng H, Xu X, Huang Z, Peng J, et al. Landscape and regulation of m6A and m6Am methylome across human and mouse tissues. Mol Cell. 2020;77(2):426-40.

36. Akichika S, Hirano S, Shichino Y, Suzuki T, Nishimasu H, Ishitani R, Sugita A, Hirose Y, Iwasaki S, Nureki O, et al. Cap-specific terminal N6-methylation of RNA by an RNA polymerase II-associated methyltransferase. Science. 2019;363(6423):eaav0080.

37. Sun $H$, Zhang M, Li K, Bai D, Yi C. Cap-specific, terminal N6-methylation by a mammalian m6Am methyltransferase. Cell Res. 2019;29(1):80-2.

38. Sendinc E, Valle-Garcia D, Dhall A, Chen H, Henriques T, Navarrete-Perea J, Sheng W, Gygi SP, Adelman K, Shi Y. PCIF1 catalyzes m6Am mRNA methylation to regulate gene expression. Mol Cell. 2019;75(3):620-30.

39. Wei J, Liu F, Lu Z, Fei Q, Ai Y, He PC, Shi H, Cui X, Su R, Klungland A, et al. Differential m6A, m6Am, and m1A demethylation mediated by FTO in the cell nucleus and cytoplasm. Mol Cell. 2018;71 (6):973-85.

40. Mauer J, Luo X, Blanjoie A, Jiao X, Grozhik AV, Patil DP, Linder B, Pickering $\mathrm{BF}$, Vasseur JJ, Chen Q, et al. Reversible methylation of m6Am in the ${ }^{\prime}$ cap controls mRNA stability. Nature. 2017;541(7637):371-5.

41. Shi $\mathrm{H}, \mathrm{Wei} \mathrm{J}, \mathrm{He} \mathrm{C}$. Where, when, and how: context-dependent functions of RNA methylation writers, readers, and erasers. Mol Cell. 2019;74(4):640-50.

42. Db DUNN. The occurrence of 1-methyladenine in ribonucleic acid. Biochim Biophys Acta. 1961;46:198-200.

43. Sharma S, Watzinger $P$, Kötter $P$, Entian KD. Identification of a nove methyltransferase, $\mathrm{Bmt2}$, responsible for the $\mathrm{N}$-1-methyl-adenosine base modification of $25 \mathrm{~S}$ rRNA in Saccharomyces cerevisiae. Nucleic Acids Res. 2013;41(10):5428-43.

44. RajBhandary UL, Stuart A, Faulkner RD, Chang SH, Khorana HG. Nucleotide sequence studies on yeast phenylalanine sRNA. Cold Spring Harb Symp Quant Biol. 1966;31:425-34.

45. Dominissini D, Nachtergaele S, Moshitch-Moshkovitz S, Peer E, Kol N, Ben-Haim MS, Dai Q, Di Segni A, Salmon-Divon M, Clark WC, et al. The dynamic N(1)-methyladenosine methylome in eukaryotic messenger RNA. Nature. 2016;530(7591):441-6.

46. Li X, Xiong X, Wang K, Wang L, Shu X, Ma S, Yi C. Transcriptome-wide mapping reveals reversible and dynamic $N(1)$-methyladenosine methylome. Nat Chem Biol. 2016;12(5):311-6.

47. Li X, Xiong X, Zhang M, Wang K, Chen Y, Zhou J, Mao Y, Lv J, Yi D, Chen $X W$, et al. Base-resolution mapping reveals distinct m1A methylome in nuclear- and mitochondrial-encoded transcripts. Mol Cell. 2017;68(5):993-1005.

48. Safra M, Sas-Chen A, Nir R, Winkler R, Nachshon A, Bar-Yaacov D, Erlacher M, Rossmanith W, Stern-Ginossar N, Schwartz S. The m1 A landscape on cytosolic and mitochondrial mRNA at single-base resolution. Nature. 2017;551(7679):251-5.

49. Ozanick S, Krecic A, Andersland J, Anderson JT. The bipartite structure of the tRNA m1A58 methyltransferase from S. cerevisiae is conserved in humans. RNA. 2005;11(8):1281-90.

50. Anderson J, Phan L, Hinnebusch AG. The Gcd10p/Gcd14p complex is the essential two-subunit tRNA (1-methyladenosine) methyltransferase of Saccharomyces cerevisiae. Proc Natl Acad Sci USA. 2000;97(10):5173-8.

51. Vilardo E, Nachbagauer C, Buzet A, Taschner A, Holzmann J, Rossmanith W. A subcomplex of human mitochondrial RNase $P$ is a bifunctional methyltransferase-extensive moonlighting in mitochondrial tRNA biogenesis. Nucleic Acids Res. 2012;40(22):11583-93.

52. Chujo T, Suzuki T. Trmt61B is a methyltransferase responsible for 1-methyladenosine at position 58 of human mitochondrial tRNAs. RNA. 2012;18(12):2269-76.

53. Liu F, Clark W, Luo G, Wang X, Fu Y, Wei J, Wang X, Hao Z, Dai Q, Zheng G, et al. ALKBH1-mediated tRNA demethylation regulates translation. Cell. 2016;167(3):816-28. 
54. Dai X, Wang T, Gonzalez G, Wang Y. Identification of YTH domaincontaining proteins as the readers for $\mathrm{N1}$-methyladenosine in RNA. Anal Chem. 2018;90(11):6380-4.

55. Motorin Y, Lyko F, Helm M. 5-Methylcytosine in RNA: detection, enzymatic formation and biological functions. Nucleic Acids Res. 2010;38(5):1415-30.

56. Squires JE, Patel HR, Nousch M, Sibbritt T, Humphreys DT, Parker BJ, Suter CM, Preiss T. Widespread occurrence of 5-methylcytosine in human coding and non-coding RNA. Nucleic Acids Res. 2012;40(11):023-33.

57. Yang X, Yang Y, Sun BF, Chen YS, Xu JW, Lai WY, Li A, Wang X, Bhattarai DP, Xiao W, et al. 5-Methylcytosine promotes mRNA export-NSUN2 as the methyltransferase and ALYREF as an $\mathrm{m} 5 \mathrm{C}$ reader. Cell Res. 2017;27(5):606-25.

58. Khoddami V, Cairns BR. Identification of direct targets and modified bases of RNA cytosine methyltransferases. Nat Biotechnol. 2013;31(5):458-64.

59. Brzezicha B, Schmidt M, Makalowska I, Jarmolowski A, Pienkowska J, Szweykowska-Kulinska Z. Identification of human tRNA:m5C methyltransferase catalysing intron-dependent $\mathrm{m} 5 \mathrm{C}$ formation in the first position of the anticodon of the pre-tRNA Leu (CAA). Nucleic Acids Res. 2006;34(20):6034-43.

60. Schaefer M, Pollex T, Hanna K, Tuorto F, Meusburger M, Helm M, Lyko F. RNA methylation by Dnmt2 protects transfer RNAs against stressinduced cleavage. Genes Dev. 2010;24(15):1590-5.

61. Fu L, Guerrero CR, Zhong N, Amato NJ, Liu Y, Liu S, Cai Q, Ji D, Jin SG, Niedernhofer $L J$, et al. Tet-mediated formation of 5-hydroxymethylcytosine in RNA. J Am Chem Soc. 2014;136(33):11582-5.

62. Yang Y, Wang L, Han X, Yang WL, Zhang M, Ma HL, Sun BF, Li A, Xia J, Chen J, et al. RNA 5-methylcytosine facilitates the maternal-tozygotic transition by preventing maternal mRNA decay. Mol Cell. 2019;75(6):1188-202.

63. Furuichi Y. Discovery of m7G-cap in eukaryotic mRNAs. Proc Jpn Acad Ser B Phys Biol Sci. 2015;91(8):394-409.

64. Zhang LS, Liu C, Ma H, Dai Q, Sun HL, Luo G, Zhang Z, Zhang L, Hu L, Dong X, et al. Transcriptome-wide mapping of internal N7-methylguanosine methylome in mammalian mRNA. Mol Cell. 2019;74(6):1304-16

65. Alexandrov A, Martzen MR, Phizicky EM. Two proteins that form a complex are required for 7 -methylguanosine modification of yeast tRNA. RNA. 2002;8(10):1253-66.

66. Jiao X, Chang J, Kilic T, Tong L, Kiledjian M. A mammalian pre-mrna 5' end capping quality control mechanism and an unexpected link of capping to pre-mrna processing. Mol Cell. 2013;50(1):104-15.

67. Zhao X, Yang Y, Sun BF, Shi Y, Yang X, Xiao W, Hao YJ, Ping XL, Chen YS, Wang WJ, et al. FTO-dependent demethylation of N6-methyladenosine regulates mRNA splicing and is required for adipogenesis. Cell Res. 2014;24(12):1403-19.

68. Chen M, Wei L, Law CT, Tsang FH, Shen J, Cheng CL, Tsang LH, Ho DW, Chiu DK, Lee JM, et al. RNA N6-methyladenosine methyltransferase-like 3 promotes liver cancer progression through YTHDF2dependent posttranscriptional silencing of SOCS2. Hepatology. 2018;67(6):2254-70.

69. Geula S, Moshitch-Moshkovitz S, Dominissini D, Mansour AA, Kol N, Salmon-Divon M, Hershkovitz V, Peer E, Mor N, Manor YS, et al. Stem cells m6A mRNA methylation facilitates resolution of naïve pluripotency toward differentiation. Science. 2015;347(6225):1002-6.

70. Tang $H$, Fan X, Xing J, Liu Z, Jiang B, Dou Y, Gorospe M, Wang W. NSun2 delays replicative senescence by repressing p27 (KIP1) translation and elevating CDK1 translation. Aging. 2015;7(12):1143-58.

71. Wang CX, Cui GS, Liu X, Xu K, Wang M, Zhang XX, Jiang LY, Li A, Yang $Y$, Lai WY, et al. METTL3-mediated m6A modification is required for cerebellar development. PLos Biol. 2018;16(6):e2004880.

72. Zhang Z, Wang M, Xie D, Huang Z, Zhang L, Yang Y, Ma D, Li W, Zhou $Q$, Yang YG, et al. METTL3-mediated N6-methyladenosine mRNA modification enhances long-term memory consolidation. Cell Res. 2018;28(11):1050-61.

73. Yoon KJ, Ringeling FR, Vissers C, Jacob F, Pokrass M, Jimenez-Cyrus D, Su Y, Kim NS, Zhu Y, Zheng L, et al. Temporal control of mammalian cortical neurogenesis by m6A methylation. Cell. 2017;171(4):877-89.
74. Li L, Zang L, Zhang F, Chen J, Shen H, Shu L, Liang F, Feng C, Chen D, Tao $\mathrm{H}$, et al. Fat mass and obesity-associated (FTO) protein regulates adult neurogenesis. Hum Mol Genet. 2017;26(13):2398-411.

75. Xu K, Yang Y, Feng GH, Sun BF, Chen JQ, Li YF, Chen YS, Zhang XX, Wang $C X$, Jiang LY, et al. Mettl3-mediated m6A regulates spermatogonial differentiation and meiosis initiation. Cell Res. 2017;27(9):1100-14.

76. Hsu PJ, Zhu Y, Ma H, Guo Y, Shi X, Liu Y, Qi M, Lu Z, Shi H, Wang J, et al. Ythdc2 is an N6-methyladenosine binding protein that regulates mammalian spermatogenesis. Cell Res. 2017;27(9):1115-27.

77. Lence T, Akhtar J, Bayer M, Schmid K, Spindler L, Ho CH, Kreim $\mathrm{N}$, Andrade-Navarro MA, Poeck B, Helm M, et al. m6A modulates neuronal functions and sex determination in Drosophila. Nature. 2016;540(7632):242-7.

78. Zhang C, Chen Y, Sun B, Wang L, Yang Y, Ma D, Lv J, Heng J, Ding Y, $X$ ue $Y$, et al. m6A modulates haematopoietic stem and progenitor cell specification. Nature. 2017;549(7671):273-6.

79. Zhao BS, Wang X, Beadell AV, Lu Z, Shi H, Kuuspalu A, Ho RK, He C. m6Adependent maternal mRNA clearance facilitates zebrafish maternal-tozygotic transition. Nature. 2017;542(7642):475-8.

80. Visvanathan A, Patil V, Arora A, Hegde AS, Arivazhagan A, Santosh V, Somasundaram K. Essential role of METTL3-mediated m6A modification in glioma stem-like cells maintenance and radioresistance. Oncogene. 2018;37(4):522-33.

81. Jin D, Guo J, Wu Y, Du J, Yang L, Wang X, Di W, Hu B, An J, Kong L, Pan L, Su G. $m^{6} A$ mRNA methylation initiated by METTL3 directly promotes YAP translation and increases YAP activity by regulating the MALAT1miR-1914-3p-YAP axis to induce NSCLC drug resistance and metastasis. J Hematol Oncol. 2019;12(1):135.

82. Dahal U, Le K, Gupta M. RNA m6A methyltransferase METTL3 regulates invasiveness of melanoma cells by matrix metallopeptidase 2. Melanoma Res. 2019;29:382-9.

83. Van Cutsem E, Sagaert X, Topal B, Haustermans K, Prenen H. Gastric cancer. Lancet. 2016;388(10060):2654-64.

84. Bijlsma MF, Sadanandam A, Tan P, Vermeulen L. Molecular subtypes in cancers of the gastrointestinal tract. Nat Rev Gastroenterol Hepatol. 2017;14(6):333-42.

85. Wang $Q$, Chen C, Ding $Q$, Zhao Y, Wang Z, Chen J, Jiang Z, Zhang $Y, X u$ $G$, Zhang J, et al. METTL3-mediated m6A modification of HDGF mRNA promotes gastric cancer progression and has prognostic significance. Gut. 2019. https://doi.org/10.1136/gutjnl-2019-319639.

86. Yue B, Song C, Yang L, Cui R, Cheng X, Zhang Z, Zhao G. METTL3mediated N6-methyladenosine modification is critical for epithelialmesenchymal transition and metastasis of gastric cancer. Mol Cancer. 2019;18(1):142.

87. Liu T, Yang S, Sui J, Xu SY, Cheng YP, Shen B, Zhang Y, Zhang XM, Yin $\mathrm{LH}, \mathrm{Pu}$ YP, et al. Dysregulated N6-methyladenosine methylation writer METTL3 contributes to the proliferation and migration of gastric cancer. J Cell Physiol. 2020;235(1):548-62.

88. Lin S, Liu J, Jiang W, Wang P, Sun C, Wang X, Chen Y, Wang H. METTL3 promotes the proliferation and mobility of gastric cancer cells. Open Med (Wars). 2019;14:25-31.

89. Zhang J, Guo S, Piao HY, Wang Y, Wu Y, Meng XY, Yang D, Zheng ZC, Zhao Y. ALKBH5 promotes invasion and metastasis of gastric cancer by decreasing methylation of the IncRNA NEAT1. J Physiol Biochem. 2019;75(3):379-89.

90. Li T, Hu PS, Zuo Z, Lin JF, Li X, Wu QN, Chen ZH, Zeng ZL, Wang F, Zheng J, et al. METTL3 facilitates tumor progression via an m6AIGF2BP2-dependent mechanism in colorectal carcinoma. Mol Cancer. 2019;18(1):112.

91. Peng W, Li J, Chen R, Gu Q, Yang P, Qian W, Ji D, Wang Q, Zhang Z, Tang $J$, et al. Upregulated METTL3 promotes metastasis of colorectal cancer via miR-1246/SPRED2/MAPK signaling pathway. J Exp Clin Cancer Res. 2019;38(1):393.

92. Deng R, Cheng Y, Ye S, Zhang J, Huang R, Li P, Liu H, Deng Q, Wu X, Lan $P$, et al. m6A methyltransferase METTL3 suppresses colorectal cancer proliferation and migration through p38/ERK pathways. Onco Targets Ther. 2019;12:4391-402.

93. Shen C, Xuan B, Yan T, Ma Y, Xu P, Tian X, Zhang X, Cao Y, Ma D, Zhu X, Zhang Y, Fang JY, Chen $\mathrm{H}$, Hong J. $\mathrm{m}^{6} \mathrm{~A}$-dependent glycolysis enhances colorectal cancer progression. Mol Cancer. 2020;19(1):72. 
94. Chen X, Xu M, Xu X, Zeng K, Liu X, Pan B, Li C, Sun L, Qin J, Xu T, He B, Pan Y, Sun H, Wang S. METTL14-mediated N6-methyladenosine modification of SOX4 mRNA inhibits tumor metastasis in colorectal cancer. Mol Cancer. 2020;19(1):106.

95. Yang X, Zhang S, He C, Xue P, Zhang L, He Z, Zang L, Feng B, Sun J, Zheng M. METTL14 suppresses proliferation and metastasis of colorectal cancer by down-regulating oncogenic long non-coding RNA XIST. Mol Cancer. 2020;19(1):46.

96. Bai Y, Yang C, Wu R, Huang L, Song S, Li W, Yan P, Lin C, Li D, Zhang Y. YTHDF1 regulates tumorigenicity and cancer stem cell-like activity in human colorectal carcinoma. Front Oncol. 2019;9:332.

97. Nishizawa Y, Konno M, Asai A, Koseki J, Kawamoto K, Miyoshi N, Takahashi H, Nishida N, Haraguchi N, Sakai D, et al. Oncogene c-Myc promotes epitranscriptome m6A reader YTHDF1 expression in colorectal cancer. Oncotarget. 2017;9(7):7476-86.

98. Ma JZ, Yang F, Zhou CC, Liu F, Yuan JH, Wang F, Wang TT, Xu QG, Zhou WP, Sun SH. METTL14 suppresses the metastatic potential of hepatocellular carcinoma by modulating N6-methyladenosine-dependent primary microRNA processing. Hepatology. 2017;65(2):529-43.

99. Chen Y, Peng C, Chen J, Chen D, Yang B, He B, Hu W, Zhang Y, Liu H, Dai $L$, et al. WTAP facilitates progression of hepatocellular carcinoma via m6A-HuR-dependent epigenetic silencing of ETS1. Mol Cancer. 2019;18(1):127.

100. Cheng X, Li M, Rao X, Zhang W, Li X, Wang L, Huang G. KIAA1429 regulates the migration and invasion of hepatocellular carcinoma by altering m6A modification of ID2 mRNA. Onco Targets Ther. 2019;12:3421-8.

101. Li J, Zhu L, Shi Y, Liu J, Lin L, Chen X. m6A demethylase FTO promotes hepatocellular carcinoma tumorigenesis via mediating PKM2 demethylation. Am J Transl Res. 2019;11(9):6084-92.

102. Rong ZX, Li Z, He JJ, Liu LY, Ren XX, Gao J, Mu Y, Guan YD, Duan YM, Zhang $X P$, et al. Downregulation of fat mass and obesity associated (FTO) promotes the progression of intrahepatic cholangiocarcinoma. Front Oncol. 2019;9:369.

103. Hou J, Zhang H, Liu J, et al. Correction to: YTHDF2 reduction fuels inflammation and vascular abnormalization in hepatocellular carcinoma. Mol Cancer. 2020;19(1):137.

104. Vincent A, Herman J, Schulick R, Hruban RH, Goggins M. Pancreatic cancer. Lancet. 2011;378(9791):607-20.

105. Taketo K, Konno M, Asai A, Koseki J, Toratani M, Satoh T, Doki Y, Mori M, Ishii H, Ogawa K. The epitranscriptome m6A writer METTL3 promotes chemo- and radioresistance in pancreatic cancer cells. Int J Oncol. 2018:52(2):621-9.

106. Xia T, Wu X, Cao M, Zhang P, Shi G, Zhang J, Lu Z, Wu P, Cai B, Miao $Y$, et al. The RNA m6A methyltransferase METTL3 promotes pancreatic cancer cell proliferation and invasion. Pathol Res Pract. 2019:215(11):152666.
107. He Y, Hu H, Wang Y, Yuan H, Lu Z, Wu P, Liu D, Tian L, Yin J, Jiang K, et al. ALKBH5 inhibits pancreatic cancer motility by decreasing long non-coding RNA KCNK15-AS1 methylation. Cell Physiol Biochem. 2018;48(2):838-46.

108. Tang B, Yang $Y$, Kang $M$, Wang $Y$, Wang $Y, B i$, , He S, Shimamoto F. $m^{6} A$ demethylase ALKBH5 inhibits pancreatic cancer tumorigenesis by decreasing WIF-1 RNA methylation and mediating Wnt signaling. Mol Cancer. 2020;19(1):3.

109. Zheng G, CoxT, Tribbey L, et al. Synthesis of a FTO inhibitor with anticonvulsant activity. ACS Chem Neurosci. 2014;5(8):658-65.

110. Yu J, Chen M, Huang H, Zhu J, Song H, Zhu J, Park J, Ji SJ. Dynamic m6A modification regulates local translation of mRNA in axons. Nucleic Acids Res. 2018;46(3):1412-23.

111. Cui $Q$, Shi $H, Y e P$, et al. $m^{6} A$ RNA methylation regulates the selfrenewal and tumorigenesis of glioblastoma stem cells. Cell Rep. 2017;18(11):2622-34.

112. Su R, Dong L, Li Y, et al. Targeting FTO suppresses cancer stem cell maintenance and immune evasion. Cancer Cell. 2020;38(1):79-96.

113. Huang Y, Su R, Sheng Y, et al. Small-molecule targeting of oncogenic FTO demethylase in acute myeloid leukemia. Cancer Cell. 2019;35(4):677-91.

114. Peng S, Xiao W, Ju D, et al. Identification of entacapone as a chemical inhibitor of FTO mediating metabolic regulation through FOXO1. Sci Transl Med. 2019;11(488):eaau7116.

115. Xu C, Liu K, Tempel W, Demetriades M, Aik W, Schofield CJ, Min J. Structures of human ALKBH5 demethylase reveal a unique binding mode for specific single-stranded N6-methyladenosine RNA demethylation. J Biol Chem. 2014;289(25):17299-311.

116. Aik W, Scotti JS, Choi H, Gong L, Demetriades M, Schofield CJ, McDonough MA. Structure of human RNA $N^{6}$-methyladenine demethylase ALKBH5 provides insights into its mechanisms of nucleic acid recognition and demethylation. Nucleic Acids Res. 2014;42(7):4741-54.

117. Elcheva IA, Wood T, Chiarolanzio K, et al. RNA-binding protein IGF2BP1 maintains leukemia stem cell properties by regulating HOXB4, MYB, and ALDH1A1. Leukemia. 2020;34(5):1354-63.

118. Mahapatra L, Andruska N, Mao C, Le J, Shapiro DJ. A novel IMP1 inhibitor, BTYNB, targets c-Myc and inhibits melanoma and ovarian cancer cell proliferation. Transl Oncol. 2017;10(5):818-27.

\section{Publisher's Note}

Springer Nature remains neutral with regard to jurisdictional claims in published maps and institutional affiliations.
Ready to submit your research? Choose BMC and benefit from:

- fast, convenient online submission

- thorough peer review by experienced researchers in your field

- rapid publication on acceptance

- support for research data, including large and complex data types

- gold Open Access which fosters wider collaboration and increased citations

- maximum visibility for your research: over 100M website views per year

At BMC, research is always in progress.

Learn more biomedcentral.com/submissions 